Supporting Information for

\title{
Strengthening and Toughening Hierarchical Nanocellulose via Humidity-Mediated Interface
}

YuanZhen Hou, ${ }^{\dagger, \S}$ Qing-Fang Guan, $\$, \S$ Jun Xia, ${ }^{\dagger} \S$ Zhang-Chi Ling, ${ }^{\ddagger}$ ZeZhou He, ${ }^{\dagger}$ ZiMeng Han, ${ }^{\sharp}$ Huai-Bin Yang, ${ }^{\ddagger}$ Ping Gu ${ }^{\dagger}{ }^{\dagger}$ YinBo Zhu, ${ }^{*} \dagger$ Shu-Hong Yu,,$*$ HengAn $\mathrm{Wu}^{*, \dagger}$

†CAS Key Laboratory of Mechanical Behavior and Design of Materials, Department of Modern Mechanics, CAS Center for Excellence in Complex System Mechanics, University of Science and Technology of China, Hefei 230027, China

Division of Nanomaterials and Chemistry, Hefei National Laboratory for Physical Sciences at the Microscale, CAS Center for Excellence in Nanoscience, Hefei Science Center of CAS, Department of Chemistry, Institute of Biomimetic Materials \& Chemistry, University of Science and Technology of China, Hefei 230026, China

$\$$ Y. Hou, Q. Guan and J. Xia contributed equally to this work.

*Corresponding authors.wwha@ustc.edu.cn (HengAn Wu); shyu@ustc.edu.cn (ShuHong Yu); zhuyinbo@ustc.edu.cn (YinBo Zhu). 

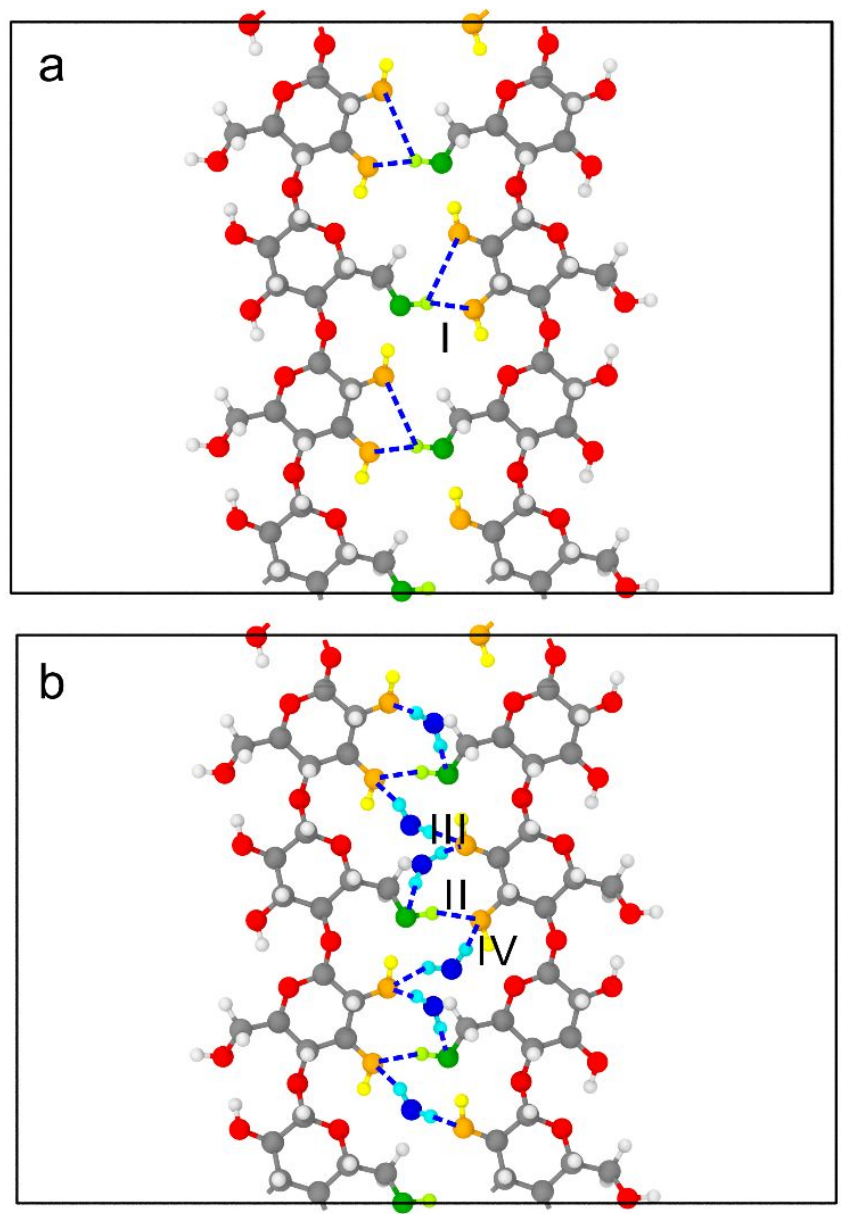

Figure S1. Optimized configurations of cellulose molecules in (010) crystal planes from DFT calculations for cases without (a) and with interfacial water molecules (b). Blue dashed lines highlight the $\mathrm{O}-\mathrm{H} \cdots \mathrm{O}$ hydrogen bonds (HBs). Water molecules are colored in blue. Gray, red, and white balls denote carbon, oxygen, and hydrogen atoms, respectively. To distinguish the hydrogen donor and acceptor at interface, hydroxyl groups in $-\mathrm{CH}_{2} \mathrm{OH}$ are colored in green while those connected with the hexagonal ring are colored in yellow. 

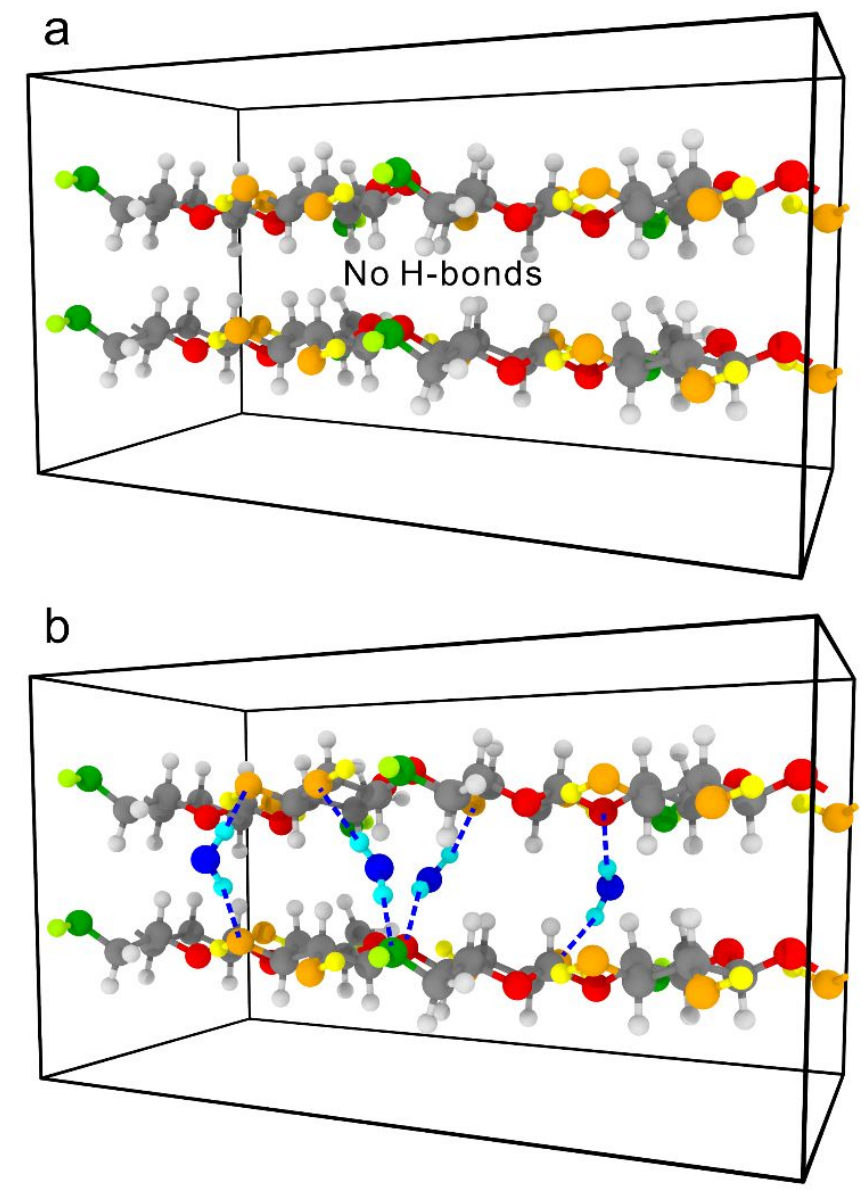

Figure S2. Optimized configurations of cellulose molecules in (100) crystal planes from DFT calculations for cases without (a) and with interfacial water molecules (b). Blue dashed lines highlight the $\mathrm{O}-\mathrm{H} \cdots \mathrm{O}$ HBs. Water molecules are colored in blue. 


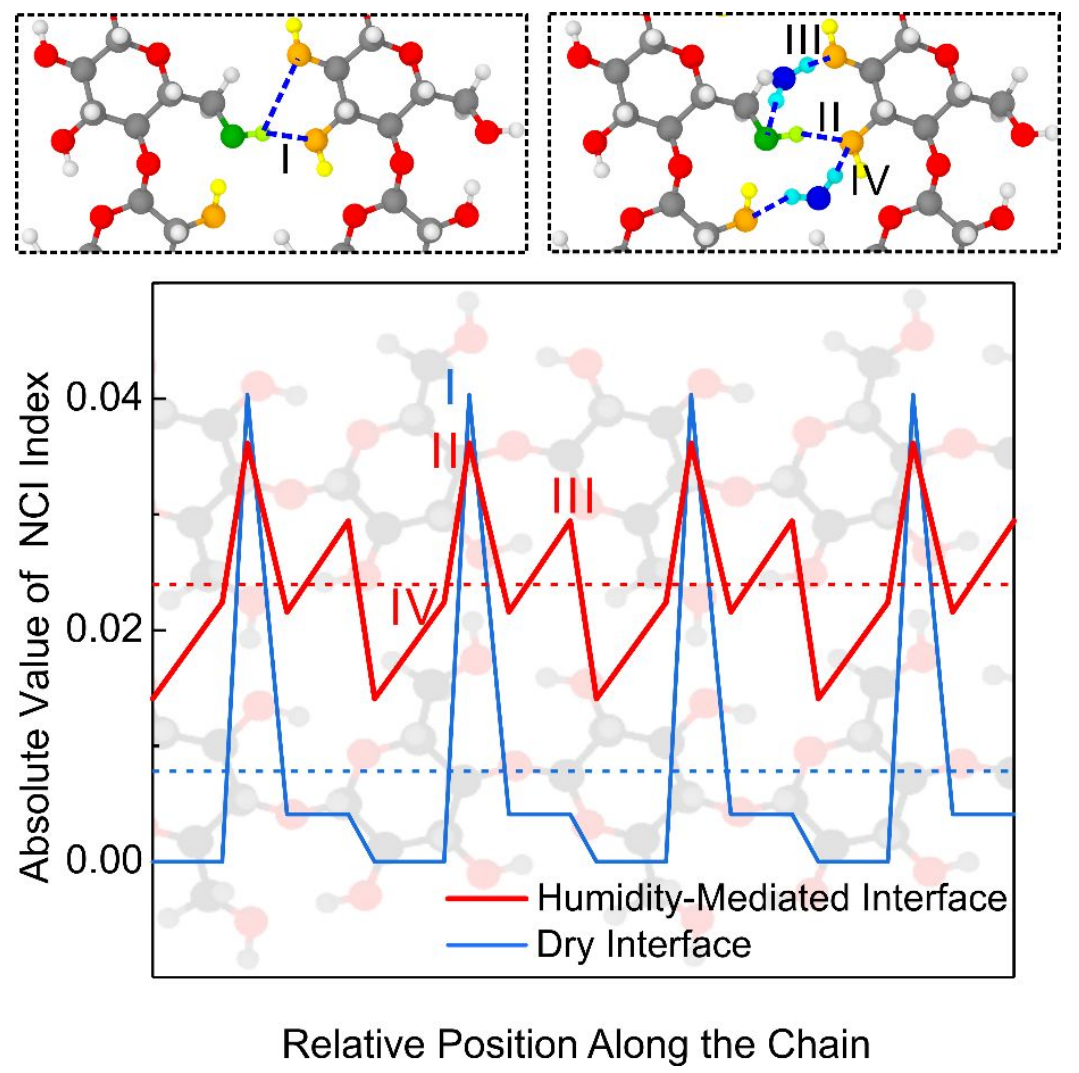

Figure S3. Plot of the NCI index value (absolute value) versus the relative position along the cellulose chain, which represents the barrier curve for the HB energy at the interface with or without interfacial water. Dashed lines represent the average value of the boundary barrier. 

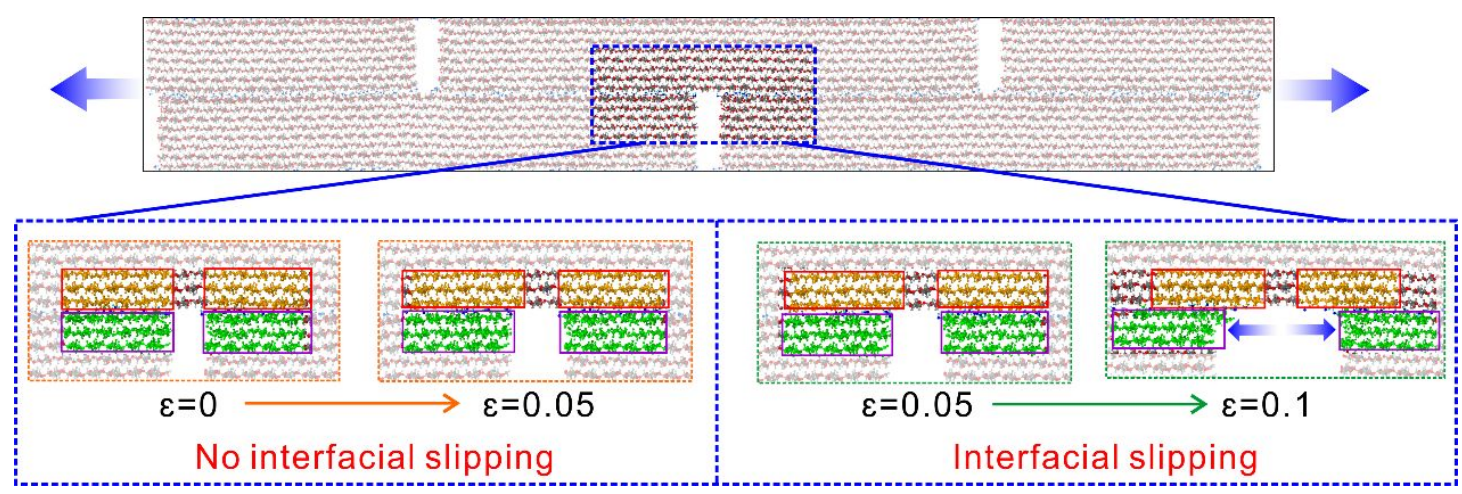

Figure S4. Local snapshots of interface changes at different tensile stages, which reflects the bilinear region in the drawing curve. In the linear-elastic stage $(\varepsilon=0 \rightarrow 0.05$, the left inset), the adjacent cellulose nanocrystals hardly slip relative to each other. The overall deformation is mainly due to the stretching of the nanocrystals themselves. In the strain-hardening stage $(\varepsilon=0.05 \rightarrow 0.1$, the right inset), an obvious interfacial slipping occurs between the adjacent cellulose nanocrystals, which dominates the overall deformation in this stage. 


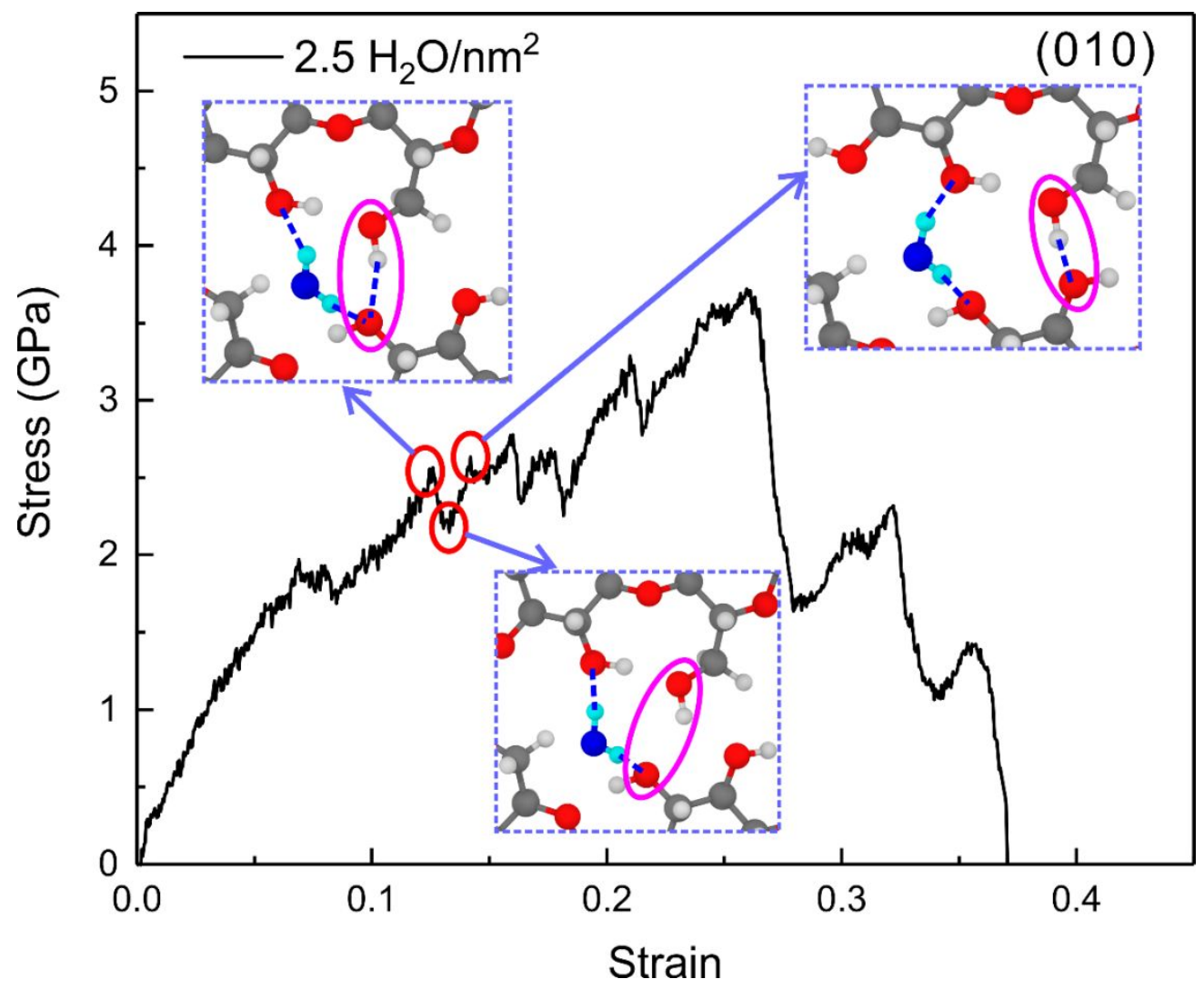

Figure S5. A schematic diagram of the fracture and reconstruction of HBs during the interfacial slipping. The insets illustrate several typical snapshots of the HB breaking and reforming. HBs between water molecules and celluloses help to maintain the overall stress level during the interfacial slipping, which reduces the amplitude of the serrated fluctuations. 

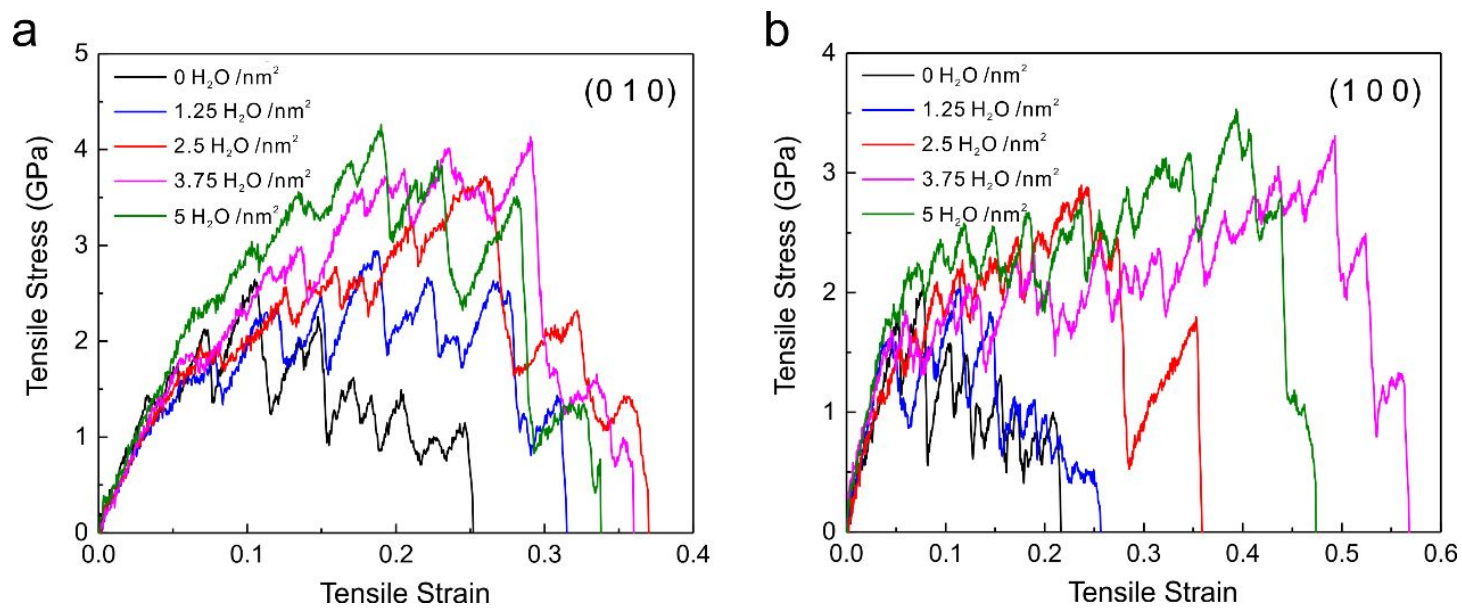

Figure S6. Tensile stress-strain curves of nanocellulose model with different interfacial water contents in (010) crystal plane (a) and in (100) crystal plane (b). The size of each cellulose nanocrystal in this model is $120 \AA \times 16.40 \AA \times 15.57 \AA$. 

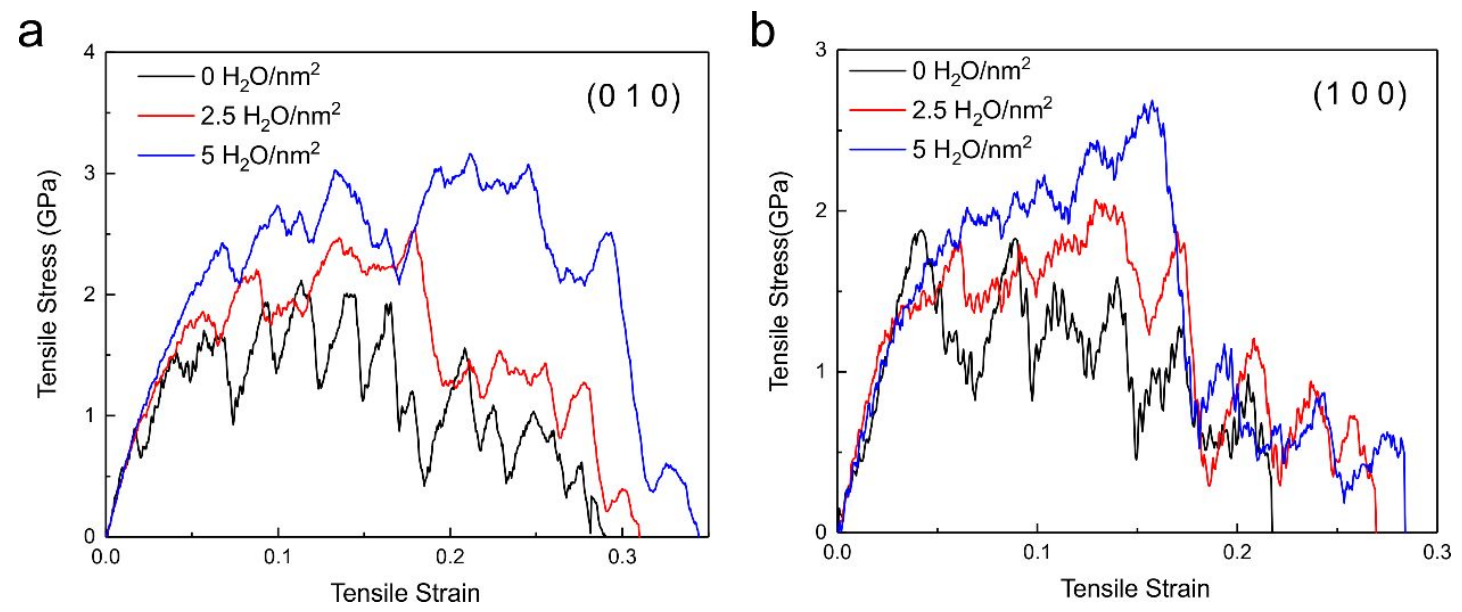

Figure S7. Tensile stress-strain curves of nanocellulose model with different interfacial water contents in (010) crystal plane (a) and in (100) crystal plane (b). The size of each cellulose nanocrystal in this model is $207.42 \AA \times 36.83 \AA \times 15.57 \AA$. 


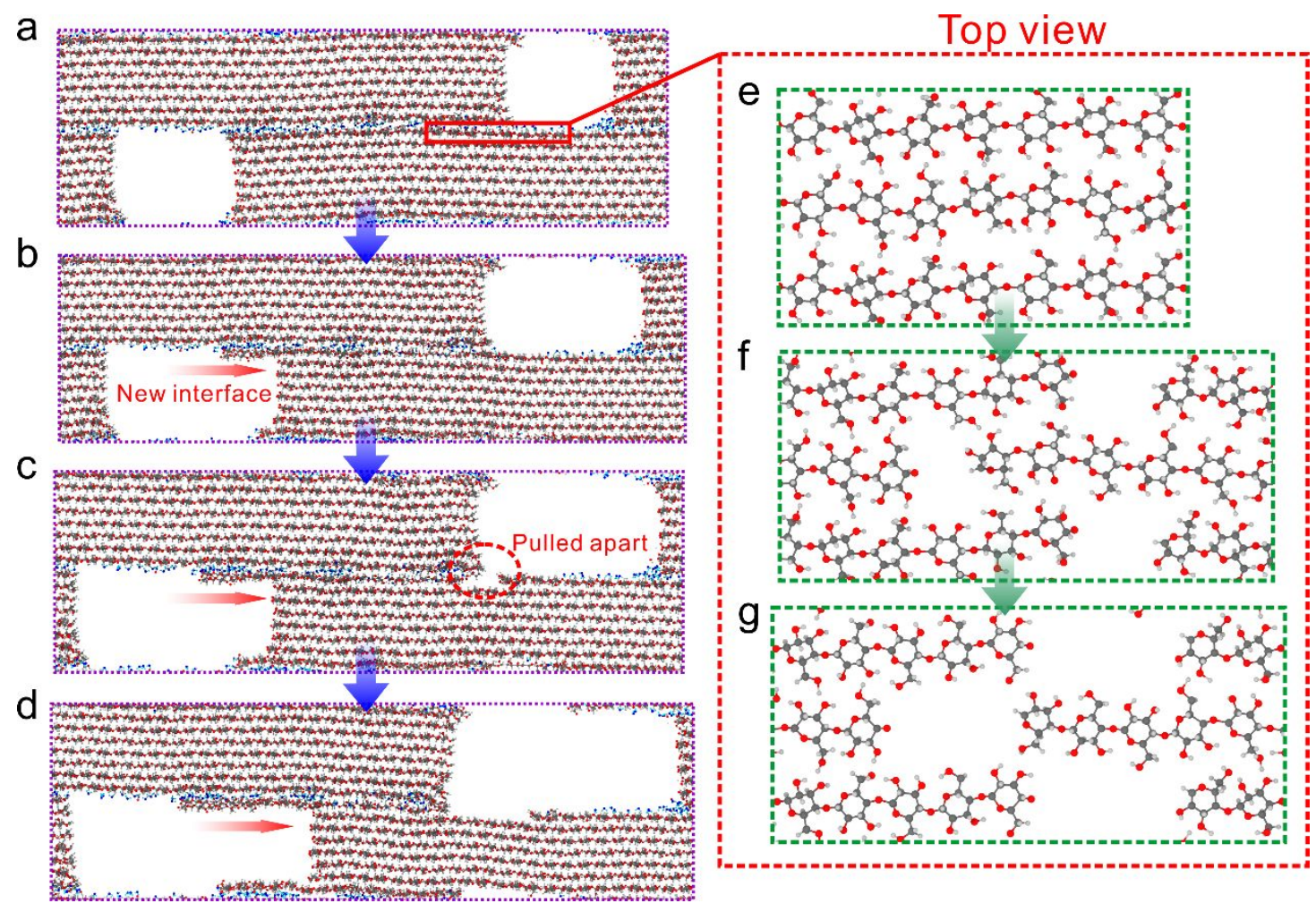

Figure S8. The formation of new interfaces when the humidity-mediated interface is slipping. From (a) to (b), the slipping interface is transferred from the CNC-Water-CNC interface to the internal interface of CNCs due to the weaker CNC-CNC HBs compared to $\mathrm{CNC}$-water-CNC $\mathrm{HBs}$ in average. The significant difference in averaged shear strength between the old and new interfaces results in the stress concentration that causes the cellulose molecular chain located on the crystal surface to be pulled apart in (c). Eventually adjacent CNCs slip along the new interface until completely pulled out in (d). (e-f) The process wherein cellulose molecular chains are pulled apart and pulled out, which contributes to the formation of new interface. 
(100)

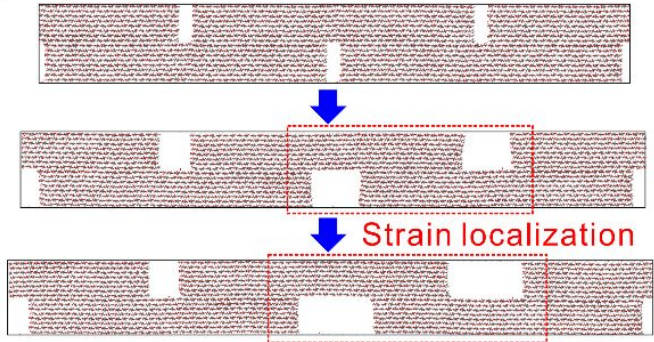

Dry Interface $\varepsilon=0$

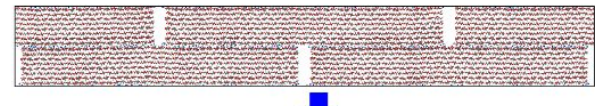

$\varepsilon=0.0875$

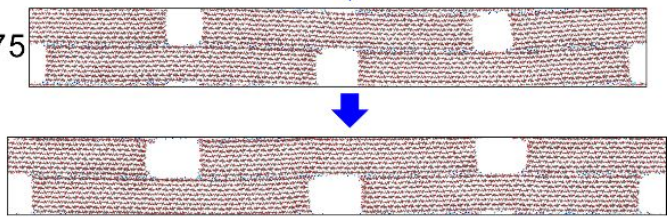

Humidity-Mediated Interface

Figure S9. Deformation modes of nanocellulose (in (100) crystal plane) without and with humidity-mediated interface during the tensile process. Red dotted boxes indicate the arising of strain localization. 

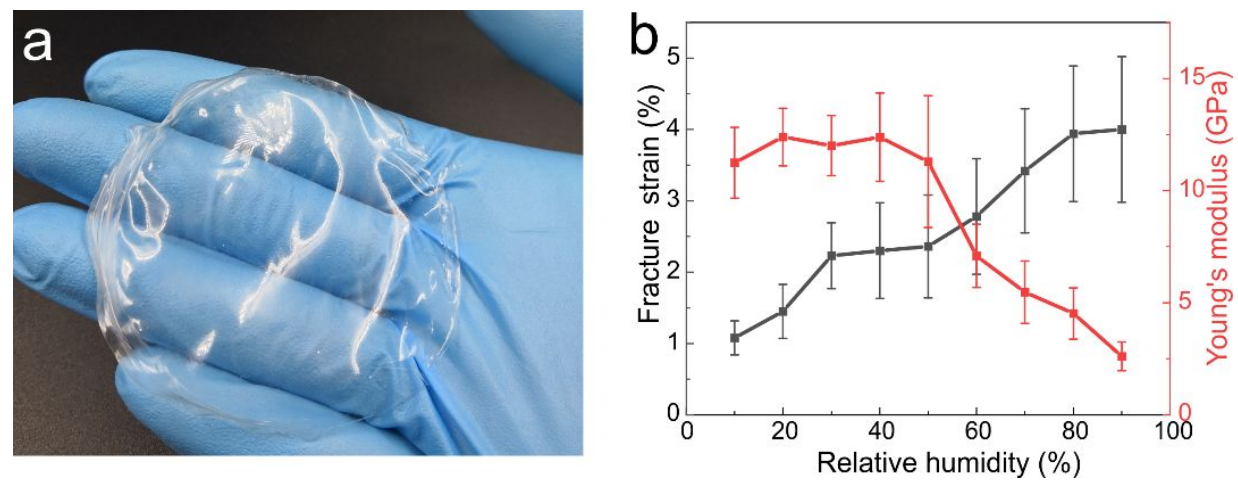

Figure S10. (a) One digital photo of the prepared CNC film. (b) Plot of the fracture strain and Young's modulus of CNC films as functions of the relative humidity. 

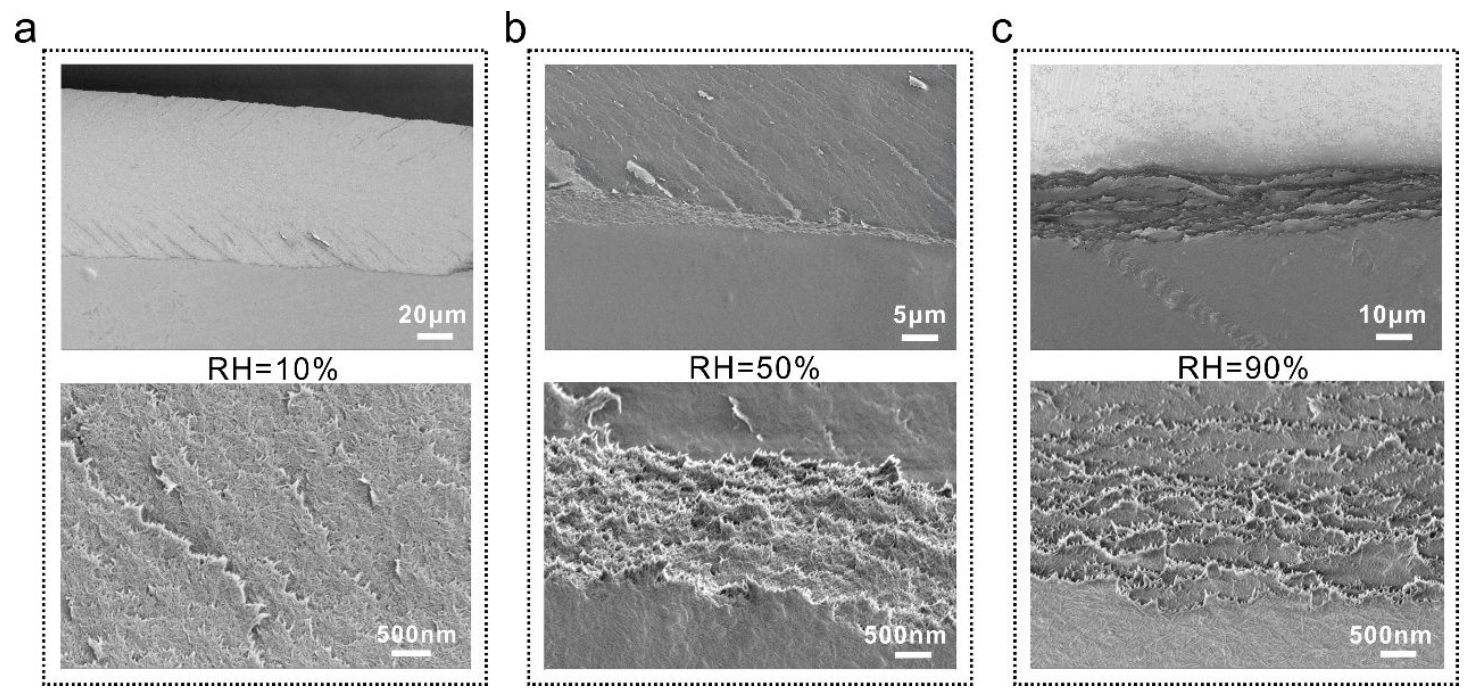

Figure S11. SEM scanning images of the fracture morphology of CNC films at $10 \%$, $50 \%$ and $90 \%$ relative humidity, respectively. The fracture morphology of CNC film under $\mathrm{RH}=10 \%$ is more smooth than that of the other two cases. For $\mathrm{RH}=50 \%$, many CNC particles are pulled out from the fracture morphology. For $\mathrm{RH}=90 \%$, layer-like fragments appear in the fracture morphology, which should be attributed to the high$\mathrm{RH}$-induced aggregation of CNCs. 